\title{
Using a Statistical-Numerical Procedure for the Selection of Pumps running as Turbines to be applied in Water Pipelines: Study Cases
}

\author{
Silvio Barbarelli ${ }^{*}$, Mario Amelio ${ }^{2}$, Gaetano Florio ${ }^{3}$ \\ ${ }^{1}$ Department of Mechanical Energy and Management Engineering, University of Calabria, \\ Via Ponte Pietro Bucci cubo 44/c, Rende, Italy \\ e-mail: silvio.barbarelli@unical.it \\ ${ }^{2}$ Department of Mechanical Energy and Management Engineering, University of Calabria, \\ Via Ponte Pietro Bucci cubo 44/c, Rende, Italy \\ e-mail: mario.amelio@unical.it \\ ${ }^{3}$ Department of Mechanical Energy and Management Engineering, University of Calabria, \\ Via Ponte Pietro Bucci cubo 44/c, Rende, Italy \\ e-mail: gaetano.florio@unical.it
}

Cite as: Barbarelli, S., Amelio, M., Florio, G., Using a Statistical-Numerical Procedure for the Selection of Pumps running as Turbines to be applied in Water Pipelines: Study Cases, J. sustain. dev. energy water environ. syst., 6(2), pp 323-340, 2018, DOI: https://doi.org/10.13044/j.sdewes.d5.0181

\begin{abstract}
A combined method using statistical and numerical models has been developed by the authors for selecting a pump running as turbine to be applied in micro-hydro plants. The data of the hydrological site chosen for the installation (head and capacity) allow the calculation of two conversion factors which identify the pump to use successfully as turbine in that place. Then, a one-dimensional model, starting from data available on the pumps manufacturers catalogues, reconstructs a virtual geometry of the pump running as turbine, and calculates the performances curves, head vs. capacity, efficiency vs. capacity, useful for identifying the operating point. Two study cases are presented to apply the proposed methodology, concerning the feasibility of the installation of a pump running as turbine in the purifier water plants of Casali and Sersale, located at 1,000 m above sea level (Calabria, South Italy).The assessment of the annual energy yield gives a confirmation of the effectiveness and convenience of using pumps running as turbines.
\end{abstract}

\section{KEYWORDS}

Hydropower, Experimental measurements, Pump as turbine, CQ-CH conversion factors, One-dimensional model, Case study.

\section{INTRODUCTION}

In the last few years, the demand for energy from renewable resources has increased more and more, taking into account the depletion of traditional sources like oil, gas and radioactive elements. Wind, sun, tides and rivers offer huge quantities of clean, green and renewable energy. Although current research lines are concentrating on local resources by favouring the smart systems approach [1], since it is the most easily used, hydraulic sources still play a great role and their exploitation is always desirable. Moreover, they have a lower environmental impact with respect to other sources [2].

\footnotetext{
${ }^{*}$ Corresponding author
} 
However, small resources, under $100 \mathrm{~kW}$, are often not considered or discarded, because of the specific cost of energy, which is higher compared to that of big hydro-electrical power plants. Traditional turbines are very expensive and their cost can reach $25 \%$ of that of the entire plant. Moreover, they need specific maintenance and have components that are scarcely available on the market. As alternative to these machines, centrifugal Pumps running As Turbines (PATs) [3] can be considered. The use of them is recommended owing to their low cost: since they are mass-produced on the market, they can cover a wide range of flow rates and heads and for this reason their cost is lower than that of a corresponding traditional hydraulic turbine. Moreover, because of the availability of spare parts even in developing countries, it is very easy and inexpensive to do the maintenance, thus saving further often mandatory costs. Even though the efficiency of a PAT is lower than a traditional turbine, it does not constitute a critical issue, above all if it is addressed to exploit energy sources otherwise wasted. Some authors estimate a capital payback period of two years or less [4] using PAT instead of traditional turbines for mini-plants in the range of 1 to $500 \mathrm{~kW}$, which is shorter than that related to the traditional turbine.

The PATs cover several applications, which involve purpose-built installations, but also existing plants managing water resources [5]. The societies, which manage the public water network, can find an interesting way to produce energy by the use of PATs: in fact, water purifier plants already have the necessary structures to create a hydraulic power plant, like intake, penstock, tubes and so on. In this case, it is very interesting to insert a hydraulic machine to recover energy otherwise lost.

But it is not easy to find the right PAT for a given application because of the lack of performances curve of the pumps on the manufacturers catalogues when they run as turbines. The question is: given a certain hydraulic resource, what kind of pump is able to provide the best energy harvesting? The problem arises because the same machine has a different behaviour when it operates as pump or as turbine. Therefore, the relation between the main parameters of the machine operating as turbine should be known, i.e., flow rate and head, and the same parameters of the machine operating instead as pump. This is due to the fact that flow rate and head of the machine operating as turbine have to be appropriate for the given application, flow rate and head of the machine operating as pump instead allow to do the choice on the manufacturer catalogue. Several methods are found in the literature: some of these are simple and aim to find the suitable pump by applying two conversion factors [6]. They express the ratio at Best Efficiency Point (BEP) between the flow rate of the turbine and that of the pump $\left(C_{\mathrm{Q}}\right)$ and the ratio at BEP between the head of the turbine and that of the pump $\left(C_{\mathrm{H}}\right)$.

These factors can be correlated in different ways. Childs [7] put them equal to the reciprocal of the pump efficiency. Sharma [8] proposed the same law, but introducing an exponentiation for both the factors. Alatorre-Frenk and Thomas [9] recommended correlations involving algebraic fractions. Stepanoff [10] put these ratios equal to the global efficiency of the pump and to the square root of the global efficiency of the pump. Hancock [11] suggested that these ratios were determined by the global efficiency of the turbine while Schmield [12] proposed a correlation linking the mentioned ratio to the hydraulic efficiency of the pump.

However, these methods give information about a single point of the machine - the BEP of the PAT - and they do not consider the issues related to the coupling machine-plant. In fact, it is necessary to know the overall characteristic head-flow rate curve of the machine and that of the site for determining the operating point of the PAT and the related efficiency.

Derakhshan and Nourbakhsh [13] propose some polynomial statistical curves, obtained by fitting measurements on a pumps sample, able to provide the information described above. Their methodology marks the way toward the problem solution, but the curves provided are strictly connected to the pump sample measured, which is poor, and need to be verified on a more consistent number of machines. 
Some other authors consider instead the possibility to adapt a PAT to the chosen site by changing its rotational speed, by trimming the impeller or by rounding the blades [14].

The present work is developed in this field, trying to fill the gap existing with the previously published research.

The final goal is to find the performance curves of a PAT suitable for a hydrological site, whose head $H_{\text {site }}$ and capacity $Q_{\text {site }}$ are known. To do this, the authors propose a procedure, which involves a statistical method and a numerical model.

The statistical method allows calculating the two conversion factors, $C_{\mathrm{Q}}$ and $C_{\mathrm{H}}$, in relation to the specific speed of the PAT working in pump mode $\left(n_{\mathrm{sp}}\right)$, which is related to the specific speed of the PAT in turbine mode $\left(n_{\mathrm{st}}\right)$, calculable with the parameters of the site, $H_{\text {site }}$ and $Q_{\text {site }}$.

Once the head and capacity at BEP of the pump suitable for the chosen site are calculated, it is possible to select suitable pumps from the composite performance chart of the manufacturer.

The authors propose a numerical model, developed during the past years [15], which is able to estimate the performance of the chosen pump working in reverse mode, as turbine (PAT).

Because the geometry of the PAT is unknown, unless it is disassembled, the code calculates the geometrical parameters of a reference pump prototype [16], starting from information deduced on the manufacturer's catalogue. Once these data are got, the code calculates the losses and determines the characteristics curves of the PAT, i.e., head vs. capacity and efficiency vs. capacity. The knowledge of these curves allows better assessment of the operating point of the plant and determination of the annual yield of energy.

Finally, a case study on possible PAT installation in the Calabrian water purifier plants of Sersale and Casali (Italy) is presented. The plants are part of the drinking water collecting and distribution system. The probability of operating off-design makes it crucial to know all the characteristic curves for estimating the yearly energy output and, in conclusion, the advisability of investing.

\section{STATISTICAL MODEL}

When a hydraulic machine has to be installed in an assigned hydrological site, its typology changes according to the covering power and also to the flow rate and to the head supplied by the site itself. In the next figure (Figure 1), the general application field of different types of traditional turbine is illustrated [6].

The same application field can be covered by PATs as illustrated in Figure 2 [6].

The use of PAT is an interesting alternative to traditional turbines because the cost of a pump is very much lower [4]. Moreover, the centrifugal pump can cover a wide range of specific speeds as illustrated in Table 1.

The necessity to have a good model able to predict the behaviour of a centrifugal pump in its reverse operating mode (such as turbine) is the main challenge in using PATs. Several theoretical/empirical correlations found in the literature try to predict the characteristic parameters head and capacity in the BEP of the PAT, involving the best head ratio and the best flow rate ratio of the same machine operating once as pump and once as turbine.

Usually, the selection of a suitable PAT for a given site starts from two conversion factors, which are:

- $C_{\mathrm{Q}}$ as the ratio of the capacity of the turbine to that of the pump, at BEP:

$$
C_{\mathrm{Q}}=\frac{Q_{\mathrm{T}}}{Q_{\mathrm{P}}}
$$


- $C_{\mathrm{H}}$ as the ratio of the two heads, defined in a similar way:

$$
C_{\mathrm{H}}=\frac{H_{\mathrm{T}}}{H_{\mathrm{P}}}
$$

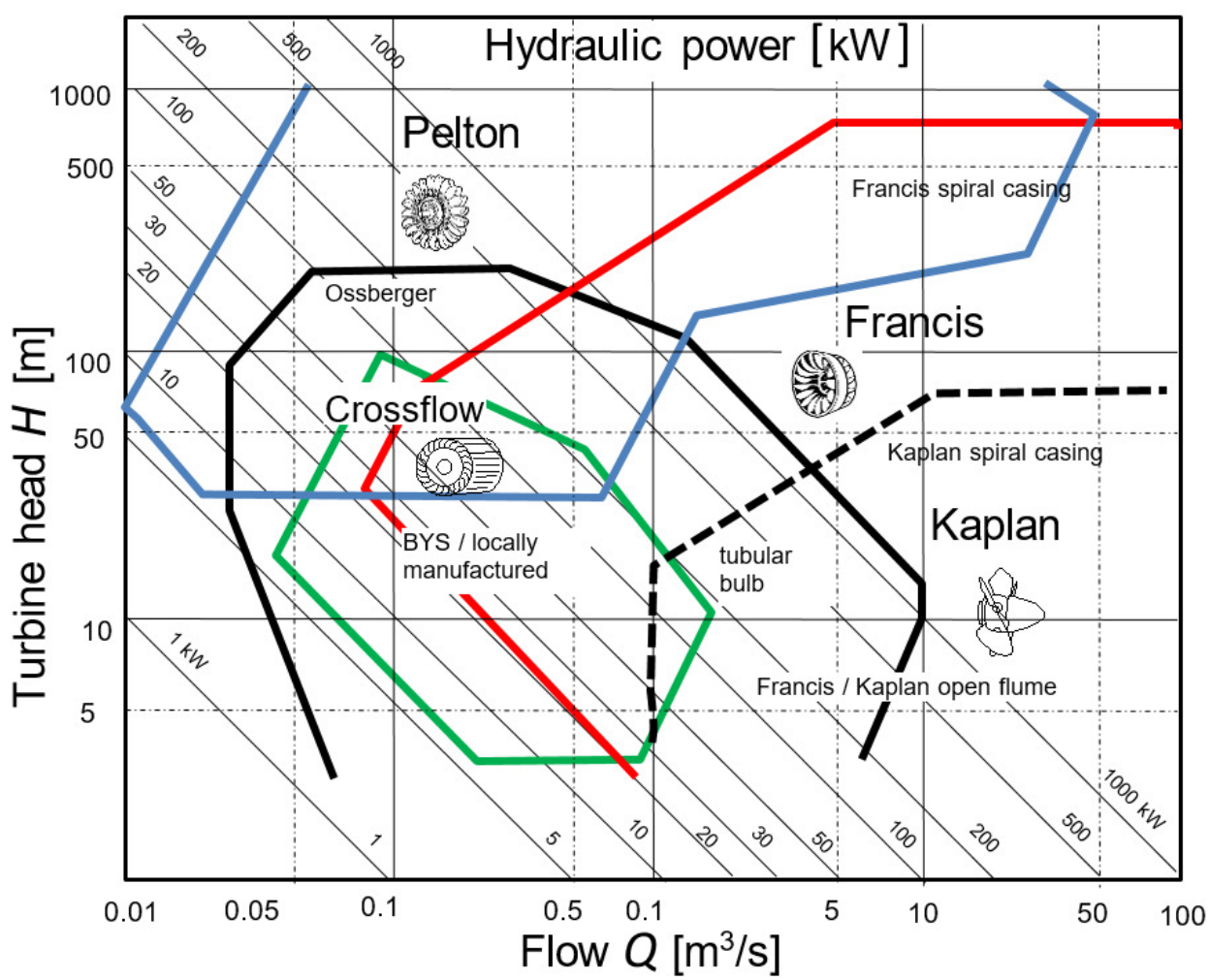

Figure 1. General range of application of different turbine types

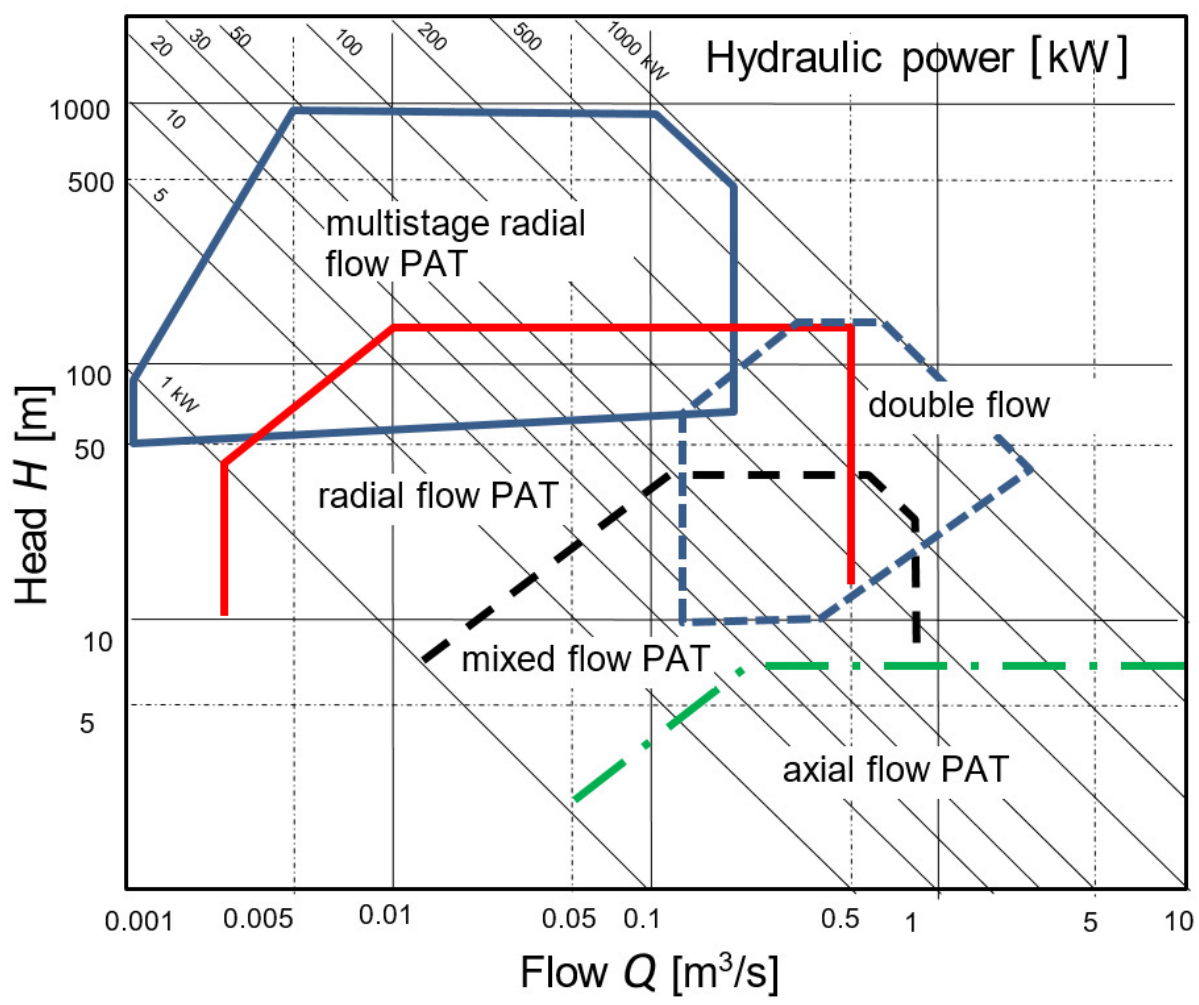

Figure 2. General range of application of different PAT types 
Table 1. Overview of basic impeller pump design

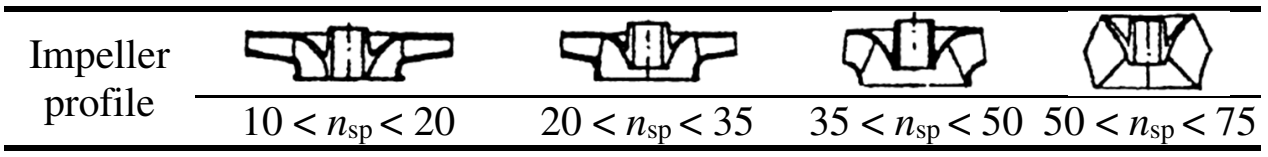

Some authors, like Grover [17] and Hergt [18], consider statistic correlations involving the specific speed of the pump by considering that the shape of the impeller, and consequently the losses typology, changes when the specific speed increases, as shown in Table 1.

The authors propose a correlation involving the specific speed of the pump $n_{\mathrm{sp}}$ defined as:

$$
n_{\mathrm{sp}}=\frac{n \sqrt{Q_{\mathrm{P}}}}{H_{\mathrm{P}}^{\frac{3}{4}}}
$$

The dependence between $C_{\mathrm{Q}}, C_{\mathrm{H}}$ and $n_{\mathrm{sp}}$ was fixed on a sample of 27 pumps. A sample of 12 pumps was measured at the hydraulic test rig of the University of Calabria (UNICAL) in direct and reverse operation [19], measurements for 4 other pumps were found in [20], while the remaining 11 in [13].

In Table 2, the values of flow, head, specific speed, efficiency at BEP point, both in direct $(\mathrm{P})$ and in reverse mode $(\mathrm{T})$, together with the conversion factors $C_{\mathrm{Q}}$ and $C_{\mathrm{H}}$, related to the pumps sample measured at UNICAL, are reported.

Table 2. Measurements at BEP point of the pumps sample of University of Calabria

\begin{tabular}{ccccccccccc}
\hline Pump & $Q_{\mathrm{P}}[1 / \mathrm{s}]$ & $H_{\mathrm{P}}[\mathrm{m}]$ & $n_{\mathrm{sp}}$ & $\eta_{\mathrm{P}}$ & $Q_{\mathrm{T}}[1 / \mathrm{s}]$ & $H_{\mathrm{T}}[\mathrm{m}]$ & $n_{\mathrm{st}}$ & $\eta_{\mathrm{T}}$ & $C_{\mathrm{Q}}$ & $C_{\mathrm{H}}$ \\
\hline Ksb 40-335 & 7.39 & 33.01 & 9.08 & 0.44 & 13.08 & 93.28 & 5.54 & 0.43 & 1.77 & 2.83 \\
Ksb 40-315 & 7.52 & 31.41 & 9.43 & 0.45 & 14.11 & 110.80 & 5.09 & 0.35 & 1.88 & 3.53 \\
Ksb 40-250 & 6.97 & 20.00 & 12.82 & 0.55 & 10.65 & 43.66 & 8.82 & 0.51 & 1.53 & 2.18 \\
Ksb 40-200 & 5.28 & 12.00 & 16.34 & 0.55 & 9.72 & 25.50 & 12.60 & 0.59 & 1.84 & 2.13 \\
Av 65-250 & 16.5 & 19.3 & 20.23 & 0.65 & 26.02 & 38 & 15.28 & 0.65 & 1.58 & 1.97 \\
Av 80-250 & 26.77 & 19.6 & 25.43 & 0.73 & 40.28 & 33.2 & 21.04 & 0.73 & 1.50 & 1.69 \\
Ksb 50-160 & 9.72 & 8.50 & 28.72 & 0.67 & 15.28 & 13.10 & 26.03 & 0.73 & 1.57 & 1.54 \\
Ksb 80-220 & 24.16 & 14.52 & 30.31 & 0.74 & 36.52 & 22.40 & 26.91 & 0.78 & 1.51 & 1.54 \\
Ksb 80-200 & 23.19 & 12.06 & 34.11 & 0.72 & 31.22 & 17.60 & 29.82 & 0.76 & 1.35 & 1.46 \\
Ksb 100-200 & 41.67 & 12.90 & 43.48 & 0.76 & 50.00 & 18.80 & 35.91 & 0.84 & 1.20 & 1.46 \\
Ksb 125-200 & 57.93 & 9.59 & 53.01 & 0.82 & 84.33 & 13.30 & 50.04 & 0.84 & 1.46 & 1.39 \\
Ksb 100-160 & 34.95 & 5.32 & 64.07 & 0.78 & 43.63 & 7.82 & 53.59 & 0.70 & 1.25 & 1.47 \\
\hline
\end{tabular}

Two simple correlations are proposed, linking the two conversion factors to the specific speed $n_{\text {sp }}$, which, in the range of value from 10 to 70, present $R$ squared value of 0.85 for $C_{\mathrm{H}}$ and 0.6 for $C_{\mathrm{Q}}$ :

$$
\begin{gathered}
C_{\mathrm{H}}=-0.000025 n_{\mathrm{sp}}^{3}+0.003615 n_{\mathrm{sp}}^{2}-0.177396 n_{\mathrm{sp}}+4.369965 \\
C_{\mathrm{Q}}=0.000221 n_{\mathrm{sp}}^{2}-0.022823 n_{\mathrm{sp}}+1.963005
\end{gathered}
$$

In the next charts (see Figure 3) the data collected for the two conversion factors on the pump sample are represented together with the interpolating curves, reporting respectively an error band of $20 \%\left(C_{\mathrm{H}}\right.$ curve) and of $15 \%\left(C_{\mathrm{Q}}\right.$ curve) which are generally acceptable for similar applications [3]. 

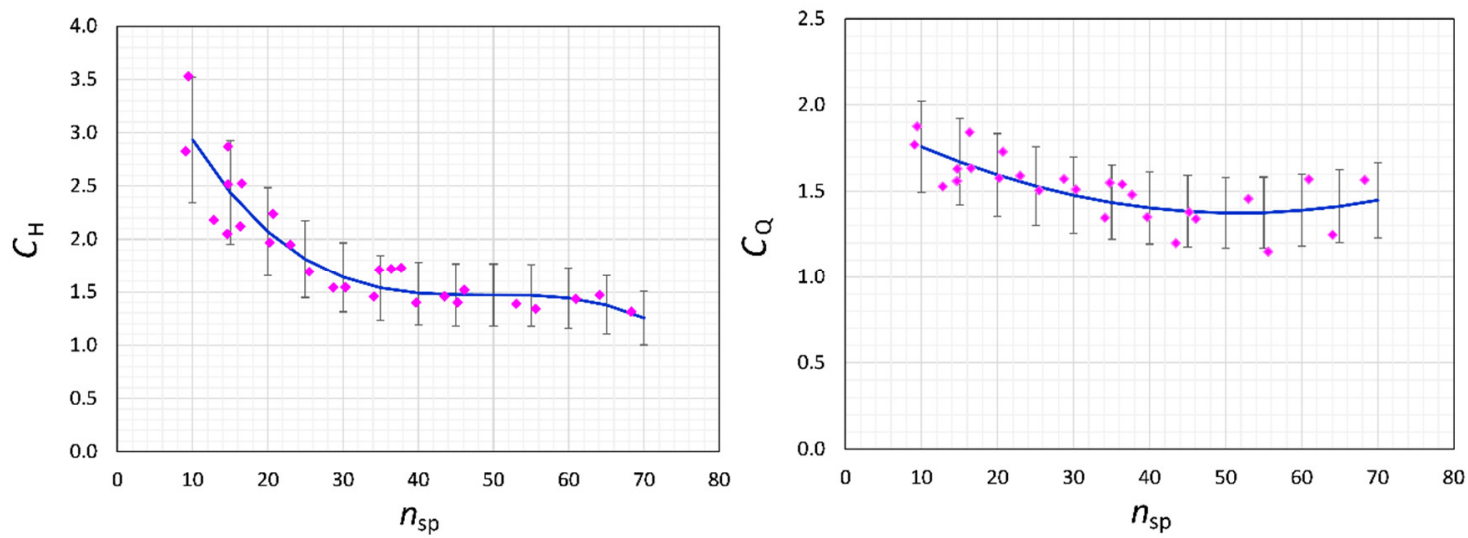

Figure 3. Interpolating curves for the conversion factors $C_{\mathrm{H}}$ and $C_{\mathrm{Q}}$

The choice of the type of correlations is purely dictated by the necessity to calculate these coefficients in a simple way, taking into account the data dispersion, the difficulty to find them and their observed trend. On the other hand, the correlations proposed by others present not dissimilar issues.

\section{Selection of the Pumps As Turbines}

The installation of a generic PAT needs the knowledge of the hydrological data of the chosen site, i.e. head $\left(H_{\text {site }}\right)$ and flow $\left(Q_{\text {site }}\right)$. These parameters allow calculation of the specific speed $\left(N_{\text {site }}\right)$ required by site defined as:

$$
N_{\text {site }}=\frac{n \sqrt{Q_{\text {site }}}}{H_{\text {site }^{\frac{3}{4}}}}
$$

which has to be matched by the specific speed available of the PAT $\left(n_{\mathrm{st}}\right)$, defined as:

$$
n_{\mathrm{st}}=\frac{n \sqrt{Q_{\mathrm{T}}}}{H_{\mathrm{T}}^{\frac{3}{4}}}
$$

since $Q_{\text {site }}$ will be equal to $Q_{\mathrm{T}}$ and $H_{\text {site }}$ will be equal to $H_{\mathrm{T}}$.

This parameter changes almost linearly with the specific speed of the pump $\left(n_{\mathrm{sp}}\right)$ and it can be correlated, from the pump sample above illustrated, as it follows:

$$
n_{\mathrm{sp}}=0.9867 n_{\mathrm{st}}+5.2818
$$

In the next figure (Figure 4) the data of the pump sample are overlapped to the above-illustrated correlation together with an error band of $5 \%$.

Figure 5 shows synthetically a flow chart illustrating the various steps. Obviously, it is necessary to know the hydrological data of the site, i.e. the head curve and the yearly frequency distribution of flow rates.

Starting from these data, it is necessary to select the design flow rate $\left(Q_{\text {site }}\right)$, determine the correspondent head $\left(H_{\text {site }}\right)$ and the specific speed required by the site $\left(N_{\text {site }}\right)$.

By considering that $N_{\text {site }}$ will be equal to $n_{\mathrm{st}}$, it is possible, by means of eq. (8), to calculate the specific speed of the pump $\left(n_{\mathrm{sp}}\right)$ and consequently to calculate the two conversion factors, $C_{\mathrm{Q}}$ and $C_{\mathrm{H}}$, by means of eqs. (4) and (5).

At this point, head and capacity of the pump at BEP can be calculated by means of the following equations: 


$$
\begin{gathered}
Q_{\mathrm{P}}=\frac{Q_{\text {site }}}{C_{\mathrm{Q}}} \\
H_{\mathrm{P}}=\frac{H_{\text {site }}}{C_{\mathrm{H}}}
\end{gathered}
$$

By using $Q_{\mathrm{P}}$ and $H_{\mathrm{P}}$ as input on the composite performance chart of the manufacturer, the pump to use as turbine can be selected.

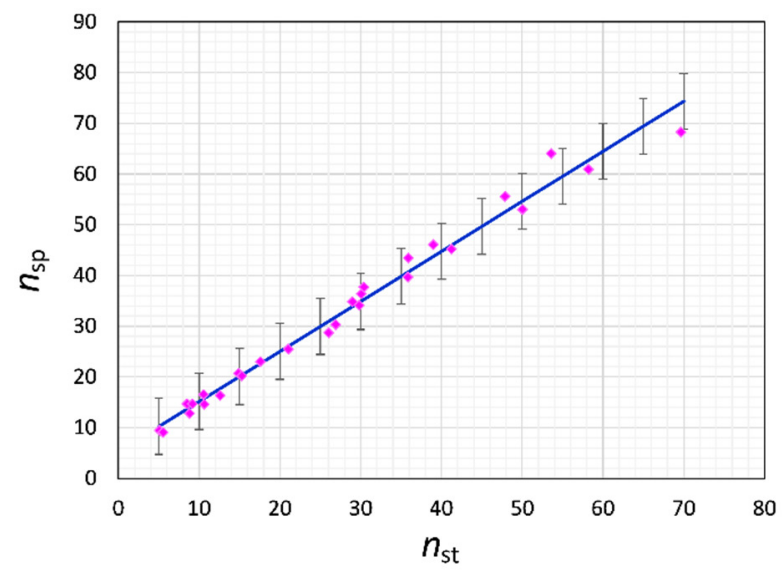

Figure 4. Interpolating curve for the specific speeds of the PAT in direct $\left(n_{\mathrm{sp}}\right)$ and in reverse $\left(n_{\mathrm{st}}\right)$ operations
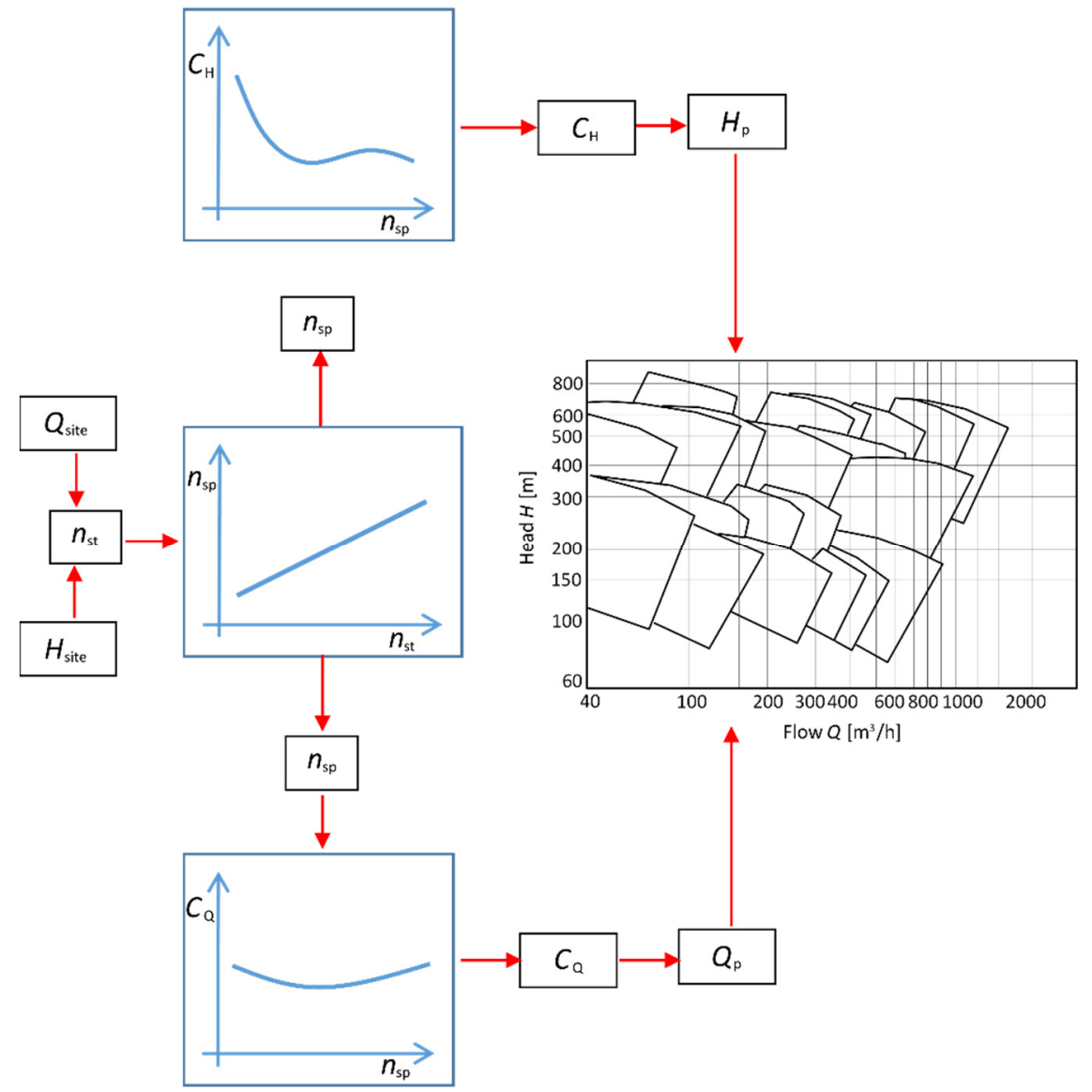

Figure 5. PAT selection procedure - flow chart 
At this point of the procedure, the pump candidate for the hydrological site is selected. With the aim to calculate the performances curve of the pump working in reverse mode it is possible to apply the UNICAL numerical model, which is widely described in [16] and synthetically reported the next section.

\section{NUMERICAL MODEL}

The authors propose a model, which can be used without knowing the geometry of the pump and which provides the performances curves of the PAT of head and efficiency by changing the entering flow. The main feature of the model proposed by the authors is its capability to operate by starting from data available in the manufacturer catalogues. It has the aim to supply extended information. In fact, it can determine all the $H(\mathrm{Q})$ and $\eta(\mathrm{Q})$ curves instead of the BEP only, by providing more accurate responses, without requiring data which are generally unavailable. Indeed, the model can determine all the useful geometrical parameters by applying standard design criteria.

In order to perform a standard design as suggested by Neumann [21] and by Lobanoff [22] it is necessary to take a precise example of centrifugal pump as a reference: a machine prototype, illustrated in Figure 3, has been chosen with a straight conical suction, rectangular volute section with linearly variable height from 0 to the throat size, and a final diffuser with square sections as a truncated pyramid (see Figure 6).

The geometry is computed by following the Lobanoff suggestions [22]. The reconstruction of the geometrical prototype can be done starting from 6 parameters that can be found in the manufacturers' catalogues: head $\left(H_{\mathrm{P}}\right)$ and flow rates $\left(Q_{\mathrm{P}}\right)$ at BEP of the pump, maximum power $\left(P_{\max }\right)$, head at the shut off $\left(H_{\mathrm{mo}}\right)$, impeller diameter $\left(D_{2}\right)$ and size of the pump $\left(y_{\mathrm{P}}\right)$.

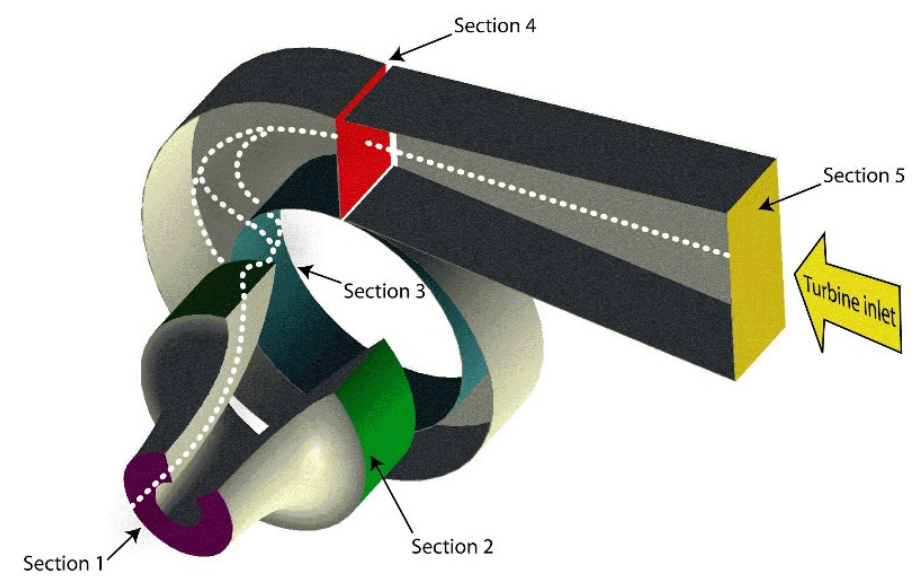

Figure 6. Reference geometry

If the geometry of the pump is unknown, the model performs the design step and it operates in "design mode".

If the geometry of the pump is known, the design step is not necessary and it can be by-passed. The model will accept, in this case, the actual geometrical parameters and it will operate in "geometry-known mode". In order to be more flexible, the design step can be performed also for a limited number of unknown geometrical parameters and so the model will operate in "mixed mode".

When the geometry of the machine is defined by applying the sizing procedure or by inputting the data known, the geometrical parameters become input data for the next calculation section of the model. Then the average velocity in each flow section and the corresponding losses can be evaluated. These are split into friction losses and dynamic losses and they are calculated by applying the following formulas: 


$$
\begin{gathered}
h_{\mathrm{f}}=\lambda \frac{c^{2}}{2 g}\left(\frac{1}{D_{\mathrm{h}}}\right) \\
h_{\mathrm{d}}=\zeta \frac{c^{2}}{2 g}
\end{gathered}
$$

The details related to the coefficients $\lambda, \zeta$ and to the geometrical passage areas are better reported in [16].

Table 3 synthetizes the main typology of losses in the various sections of the machine. The dynamic losses are calculated as Idel'cick [23] suggests. The volute has been divided in sectors for better applying eq. (11) [16].

Table 3. Hydraulic losses typology

\begin{tabular}{ccc}
\hline Section & \multicolumn{2}{c}{ Losses typology } \\
\hline Sect. 5 - diffuser & Friction losses & Dynamic losses \\
Sect. 4 - volute & Friction losses calculated by dividing the volute in sectors \\
Sect. 3 - vaneless & Friction losses & Dynamic losses \\
& Diffusion losses & Shock losses \\
Sect. 2 - impeller & Friction losses & Sudden restriction losses \\
& Drag losses & Discharge losses \\
Sect. $1-$ discharge & \multicolumn{2}{c}{} \\
\hline
\end{tabular}

Once the losses have been calculated, the real head $\left(H_{\mathrm{m}}\right)$ can be expressed as a sum of the Eulerian work $\left(H_{\mathrm{th}}\right)$ and of the losses themselves.

The flow chart illustrated in Figure 7 summarizes the whole process that begins by setting the initial value ( $\left.Q_{\text {iteration }}\right)$ of the capacity. This value has to take into account the leakages through clearances and then the volumetric efficiency $\left(\eta_{\mathrm{v}}\right)$. For this reason, the procedure is iterative.

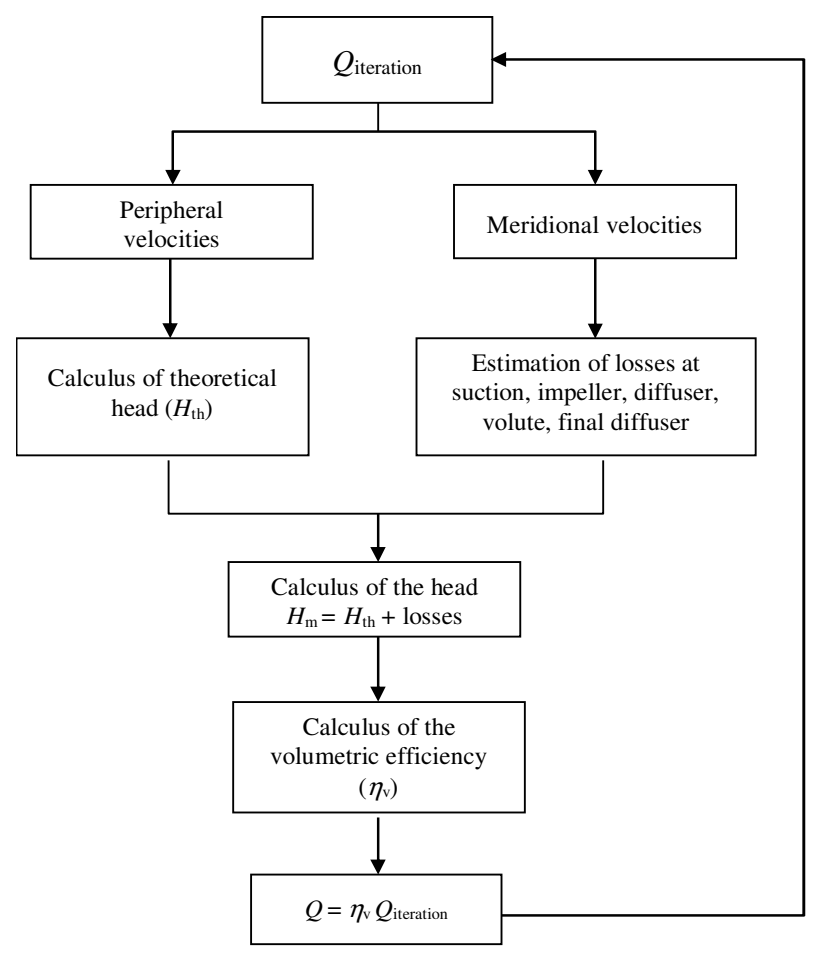

Figure 7. Head calculus flow chart 
Finally, once $H_{\mathrm{m}}$ is calculated, the hydraulic efficiency can be calculated and, taking into account the volumetric efficiency $\left(\eta_{\mathrm{v}}\right)$ and the disc efficiency $\left(\eta_{\mathrm{D}}\right)$, the power output of the PAT and the total efficiency of the PAT will be calculated as follows:

$$
\begin{gathered}
P=\eta_{\mathrm{H}} \eta_{\mathrm{v}} \eta_{\mathrm{D}} \rho g Q H \\
\eta_{\mathrm{tot}}=\eta_{\mathrm{H}} \eta_{\mathrm{v}} \eta_{\mathrm{D}}
\end{gathered}
$$

\section{STUDY CASES}

The two Calabrian sites, chosen for recovering energy with PATs, are the water purifier plants of Casali and Sersale. Each site is characterized by variable flow rates, according to the requirements of the users. In the next figures, the schemes of the water purifier plants of Casali (Figure 8) and Sersale (Figure 9) are reported. In these figures, the proposed installations are also recorded in the dashed red circle.

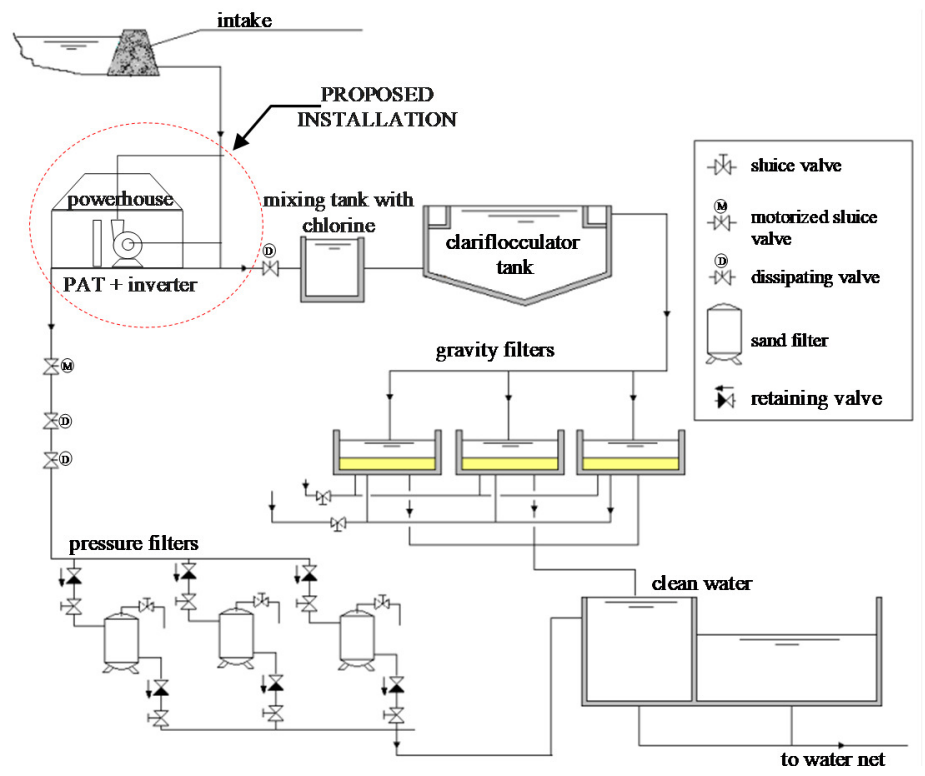

Figure 8. Water purifier plant of Casali and proposed installation

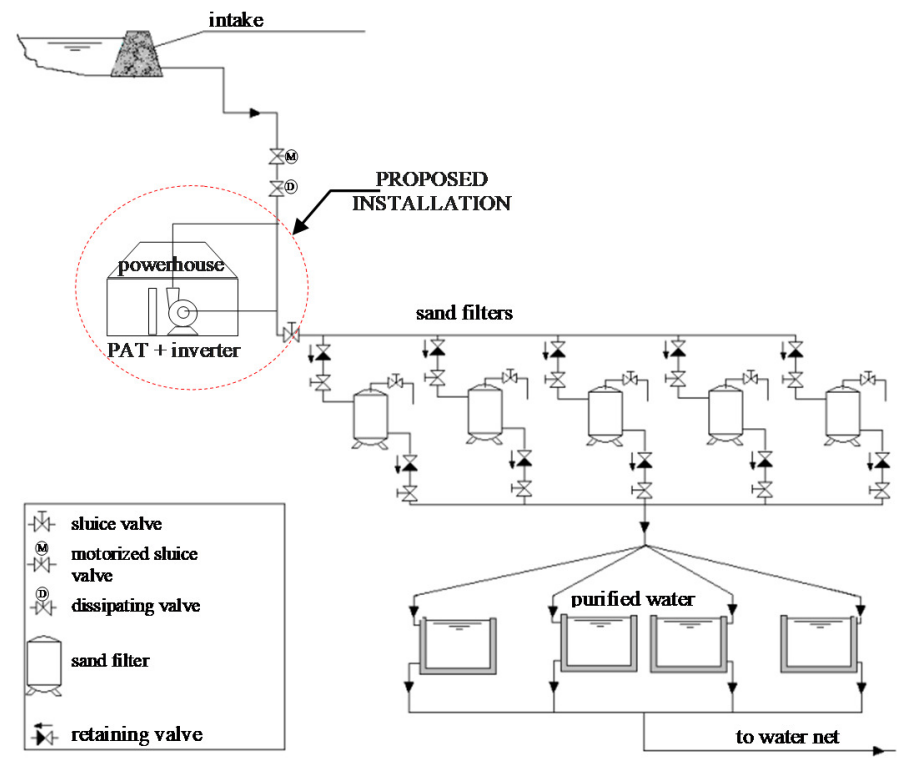

Figure 9. Water purifier plant of Sersale and proposed installation 
The two sites, located at over 1,000 $\mathrm{m}$ above sea level, are characterized by variable flow rates, according to the users' needs. In the next figures [Figure 10 (Casali) and Figure 11 (Sersale)] the characteristic head curves of the penstocks are illustrated together with the yearly distributions of the flow rates.

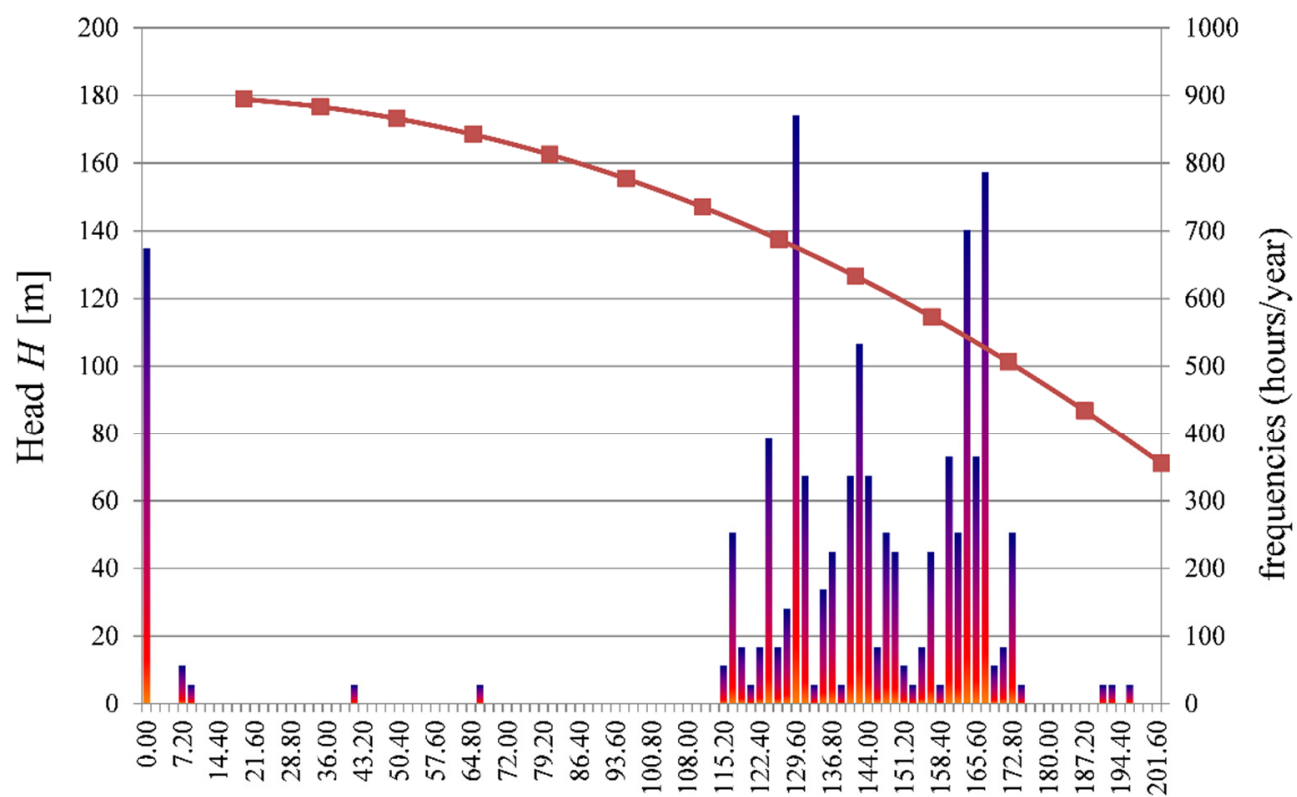

Flow $Q\left[\mathrm{~m}^{3} / \mathrm{h}\right]$

Figure 10. Head characteristic curve of the penstock and yearly frequencies of flow rates of Casali site

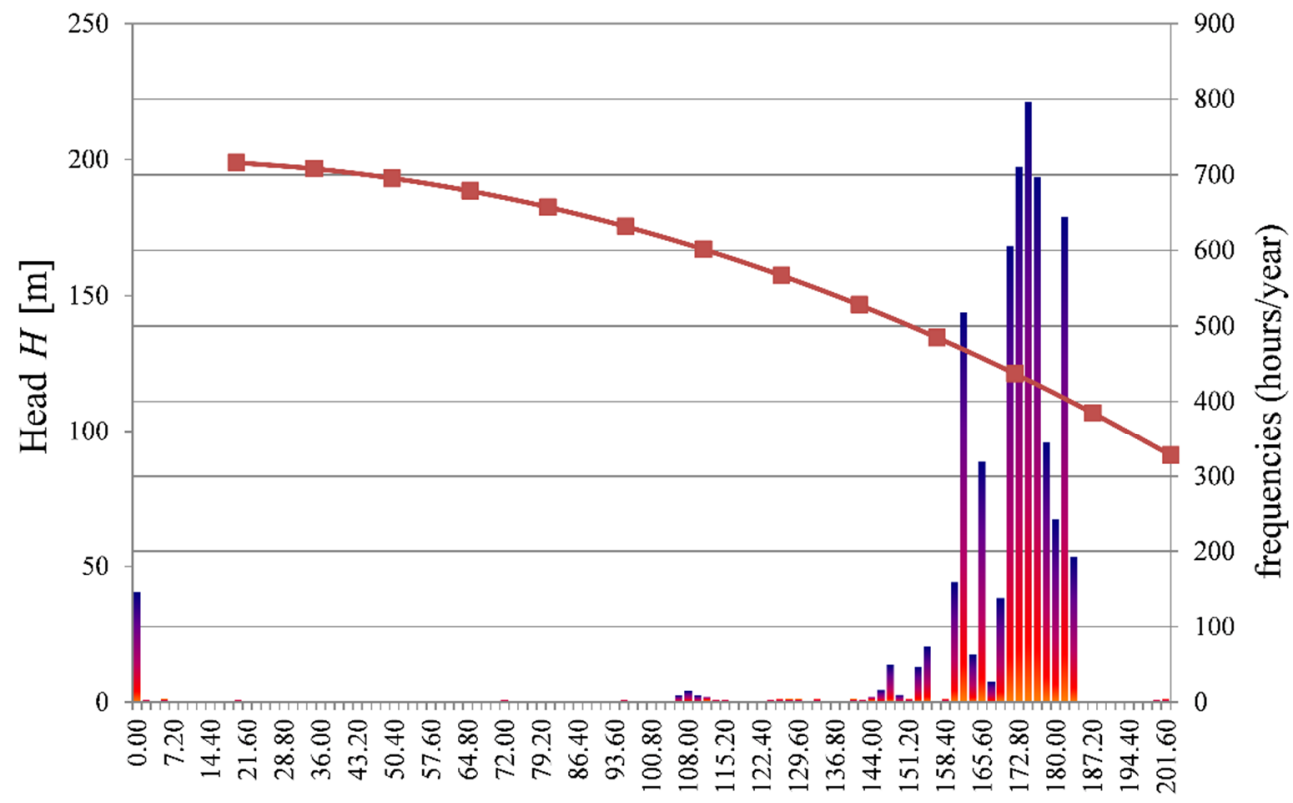

Flow $Q\left[\mathrm{~m}^{3} / \mathrm{h}\right]$

Figure 11. Head characteristic curve of the penstock and yearly frequencies of flow rates of Sersale site

The most frequent flow rates of the Casali plant (see Figure 10) vary in the range of 115 to $172 \mathrm{~m}^{3} / \mathrm{h}$, while the related heads vary from $145 \mathrm{~m}$ to $100 \mathrm{~m}$, whereas the most frequent flow rates of the Sersale plant (see Figure 11) vary in the range of 144 to 185 $\mathrm{m}^{3} / \mathrm{h}$, while the related heads vary from $145 \mathrm{~m}$ to $106 \mathrm{~m}$. 


\section{SELECTION OF THE PATS}

Starting from the hydrological data of the two sites and applying the procedure illustrated in Figure 5, as well as the eqs. from (3) to (9), it is possible to select the suitable PATs. In Table 4 the results of the selection are reported. The procedure refers to the average values of the above-illustrated flow rates ranges. The flow rate of $143.5 \mathrm{~m}^{3} / \mathrm{h}$ is the mean value considered for the Casali site whose head of $123.4 \mathrm{~m}$ corresponds (see Figure 10), while the flow rate of $164.5 \mathrm{~m}^{3} / \mathrm{h}$ is the mean value considered for the Sersale site whose head of $126.2 \mathrm{~m}$ corresponds (see Figure 11). In these conditions, the specific speeds of the sites, calculated taking into account a rotational speed of 2,950 rpm, result 15.91 for Casali and 16.75 for Sersale. By applying eq. (8), the specific speeds of the pump candidate for the sites can be determined. By applying eqs. (4) and (5), these values allow calculation of the conversion factors $C_{\mathrm{Q}}$ and $C_{\mathrm{H}}$, and finally, by applying eqs. (9) and (10), the flow rates and heads at BEP of the candidate pumps (see Table 4).

Table 4. Selecting PAT results

\begin{tabular}{ccc}
\hline & Casali & Sersale \\
\hline$Q_{\text {site }}\left[\mathrm{m}^{3} / \mathrm{h}\right]$ & 143.5 & 164.5 \\
$H_{\text {site }}[\mathrm{m}]$ & 123.4 & 126.2 \\
$N_{\text {site }}\left[\mathrm{rpm} \mathrm{m} \mathrm{rm} \mathrm{s}^{3 / 4} \mathrm{~s}^{1 / 2}\right]$ & 15.91 & 16.75 \\
$n_{\text {st }}\left[\mathrm{rpm} \mathrm{m} \mathrm{m}^{3 / 4} \mathrm{~s}^{1 / 2}\right]$ & 15.91 & 16.75 \\
$n_{\mathrm{sp}}\left[\mathrm{rpm} \mathrm{m} \mathrm{sm}^{3 / 4} \mathrm{~s}^{1 / 2}\right]$ & 20.5 & 21.33 \\
$C_{\mathrm{H}}$ & 1.95 & 1.90 \\
$C_{\mathrm{Q}}$ & 1.57 & 1.56 \\
$Q_{\mathrm{P}}\left[\mathrm{m}^{3} / \mathrm{h}\right]$ & 91.29 & 105.50 \\
$H_{\mathrm{P}}[\mathrm{m}]$ & 63.21 & 66.43 \\
Choice of the PAT & $65-250$ & $65-250$ \\
\hline
\end{tabular}

In Figure 12 it is possible to see how the pump candidate for the two sites is the same, i.e. the 65-250 pump. For applying the numerical model it is possible to extract the input depicted in the following table (Table 5) from the manufacturer catalogue.

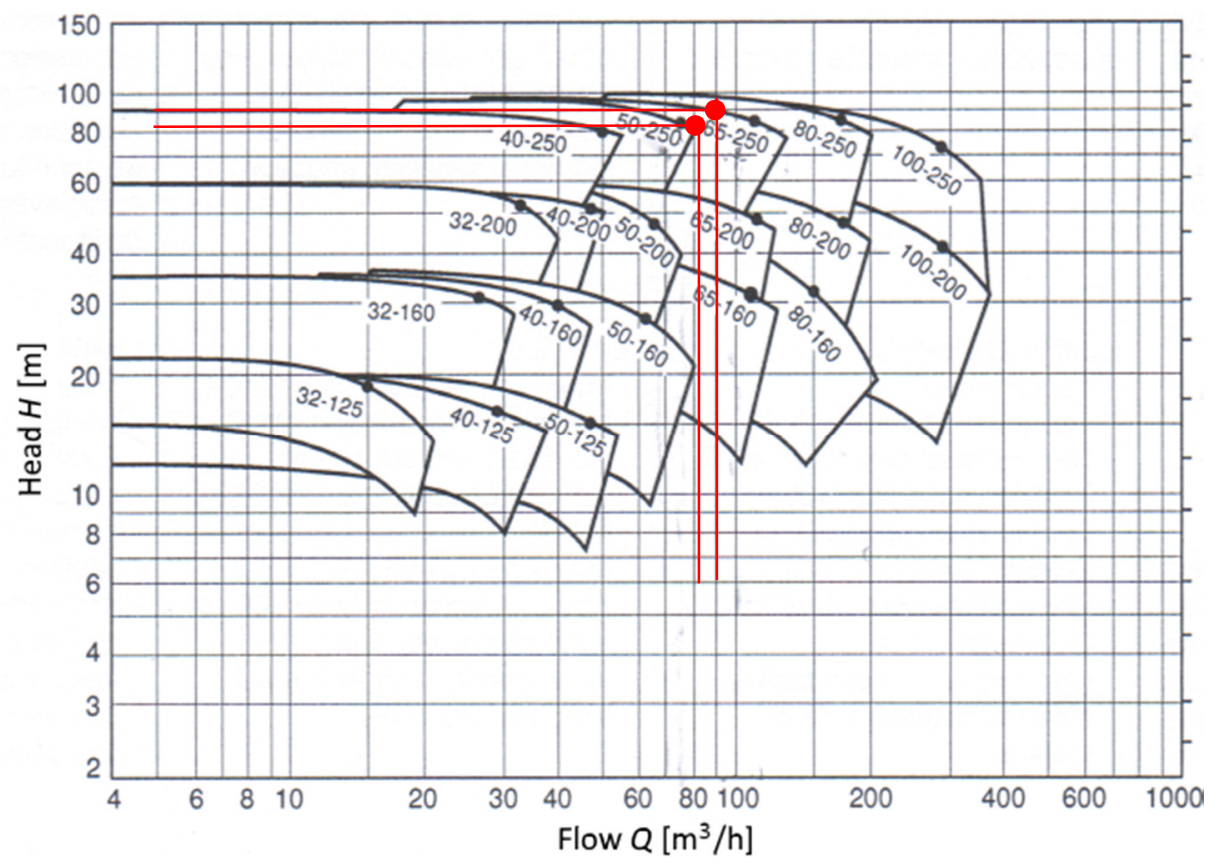

Figure 12. Choice of the pump on the composite performance chart of the manufacturer 
Table 5. Input of the model

\begin{tabular}{cc}
\hline$D_{2}[\mathrm{~mm}]$ & 226 \\
$y_{\mathrm{P}}[\mathrm{mm}]$ & 250 \\
$Q_{\mathrm{P}}\left[\mathrm{m}^{3} / \mathrm{h}\right]$ & 105 \\
$H_{\mathrm{P}}[\mathrm{m}]$ & 60 \\
$H_{\mathrm{mo}}[\mathrm{m}]$ & 70 \\
$P_{\max }[\mathrm{kW}]$ & 55 \\
$\mathrm{rpm}\left[\mathrm{min}^{-1}\right]$ & 2,950 \\
\hline
\end{tabular}

Figures $13 \mathrm{a}$ and $13 \mathrm{~b}$ report the performances curves foreseen from the UNICAL model.

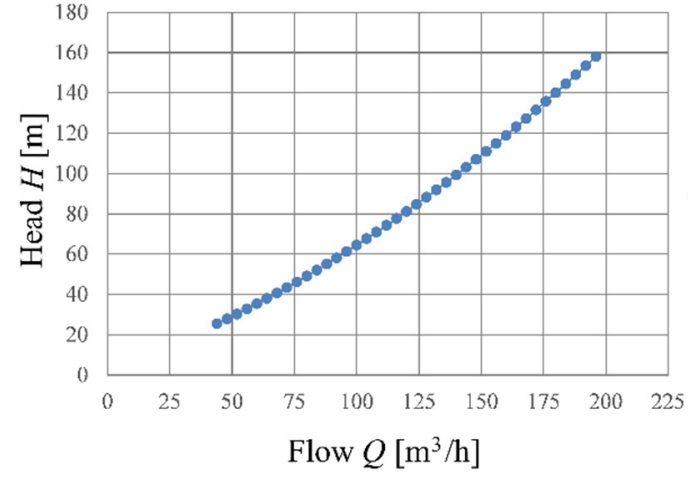

Figure 13a. Head foreseen

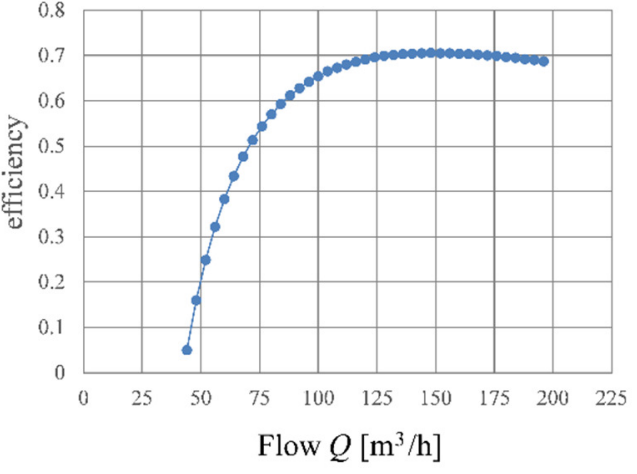

Figure 13b. Efficiency foreseen

The curves provided by the software give a lot of information: in fact, by coupling the head curve of the PAT with the head curve of the site, the operating point can be calculated, and thus the correspondent efficiency. The designer can be satisfied, if the efficiency is that expected as maximum value, or a little lower.

Figure 14 reports the characteristic curves of the PAT (head and efficiency vs. flow rate) and the head curves of the sites. One can observe that the two operating points are characterised by efficiency close to the maximum value of 0.7 and that confirms the goodness of the choice.

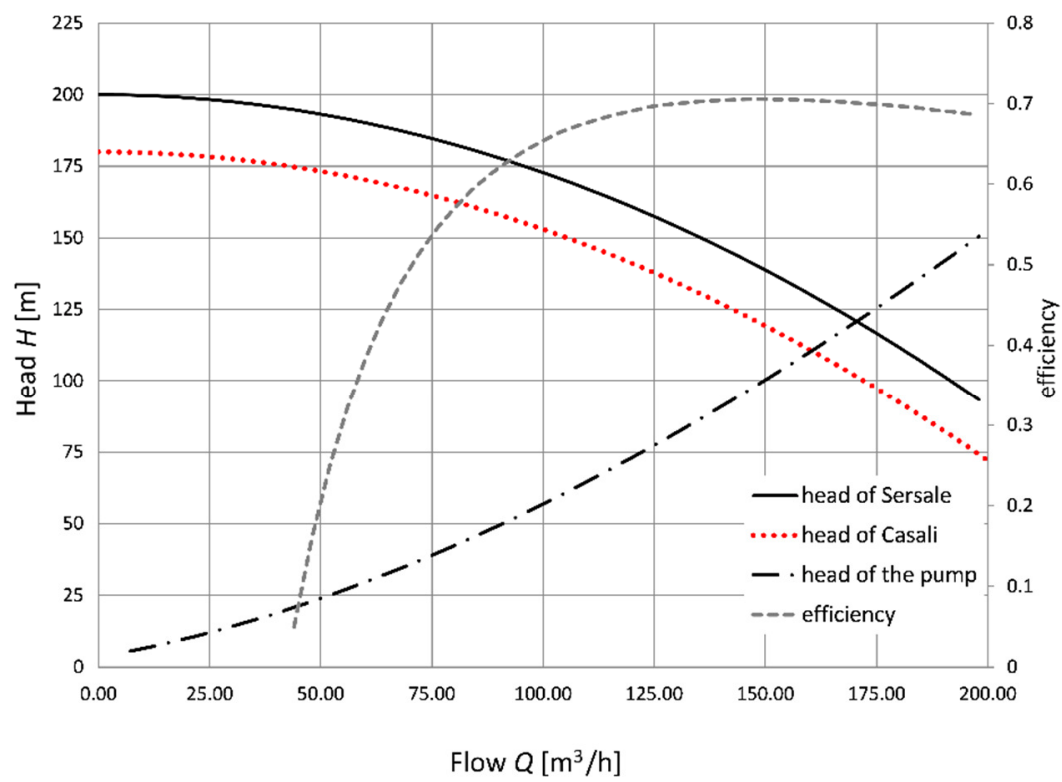

Figure 14. Characteristic curves - operating points 
All the parameters connected to the two operating points are reported in Table 6 , where the powers are estimated as follows:

$$
P=\rho g \eta_{\mathrm{el}} \eta_{\mathrm{T}} Q_{\mathrm{T}} H_{\mathrm{T}}
$$

Table 6. Operating points of the selected PAT

\begin{tabular}{ccc}
\hline & Casali & Sersale \\
\hline$Q_{\mathrm{T}}\left[\mathrm{m}^{3} / \mathrm{h}\right]$ & 160 & 170 \\
$H_{\mathrm{T}}[\mathrm{m}]$ & 110 & 120 \\
$\eta_{\mathrm{T}}$ & 0.7 & 0.7 \\
$\eta_{\mathrm{el}}$ & 0.96 & 0.96 \\
$P[\mathrm{~kW}]$ & 32.2 & 37.3 \\
\hline
\end{tabular}

The PAT obviously has to work off-design for all the time since the flow rates are variable in the ranges of values above described. It was anyway observed that in the overall range of values common to both the sites, i.e. from 115 to $185 \mathrm{~m}^{3} / \mathrm{h}$, the PAT achieves almost constant efficiencies, changing from $68 \%$ to $70 \%$. This is the advantage of working with PATs, since efficiencies are little variable, above all to the right of the BEP.

\section{OFF-DESIGN OPERATIONS}

The selected PAT always operates off-design because of the changeable flow rates. In the next scheme, illustrated in Figure 15, how to manage these conditions is illustrated. When the flow rate is lower than that of design (see Figure 16) the PAT will work with a head lower than that of the site. In this case, a valve, located at discharge of the PAT, will dissipate the surplus of the head.

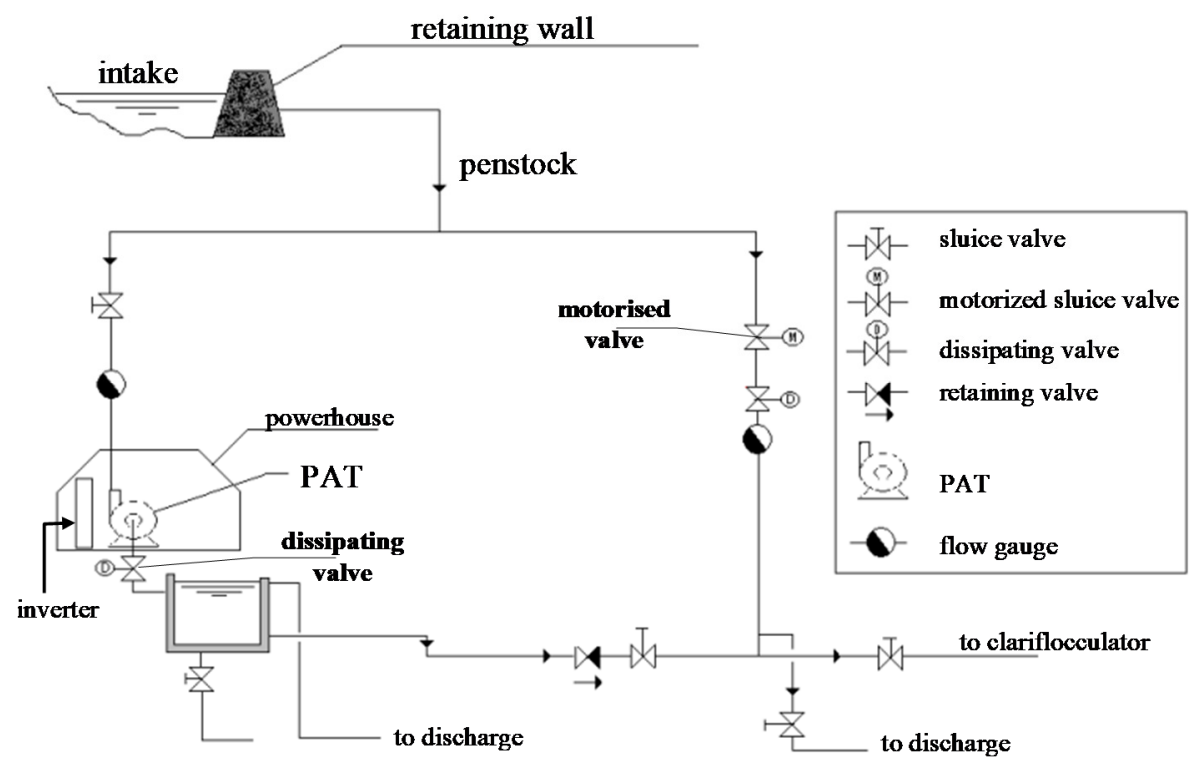

Figure 15. Scheme of the purifier plant with PAT

When the flow rate instead is higher than that of design (see Figure 17) the PAT, having to work with the same head as the site, will have to receive a lower flow rate. In this case, a motorized valve, located on a bypass pipe, will allow the passage of the excess flow rate. 
Taking into account the above described operations in off design conditions, the next tables (Tables 7 and 8) report an extract of the data related to the entire variation field of the flow rates coming from the water purifiers and the data of the same PAT 65-250, selected for both the site of Casali (Table 7) and of Sersale (Table 8).

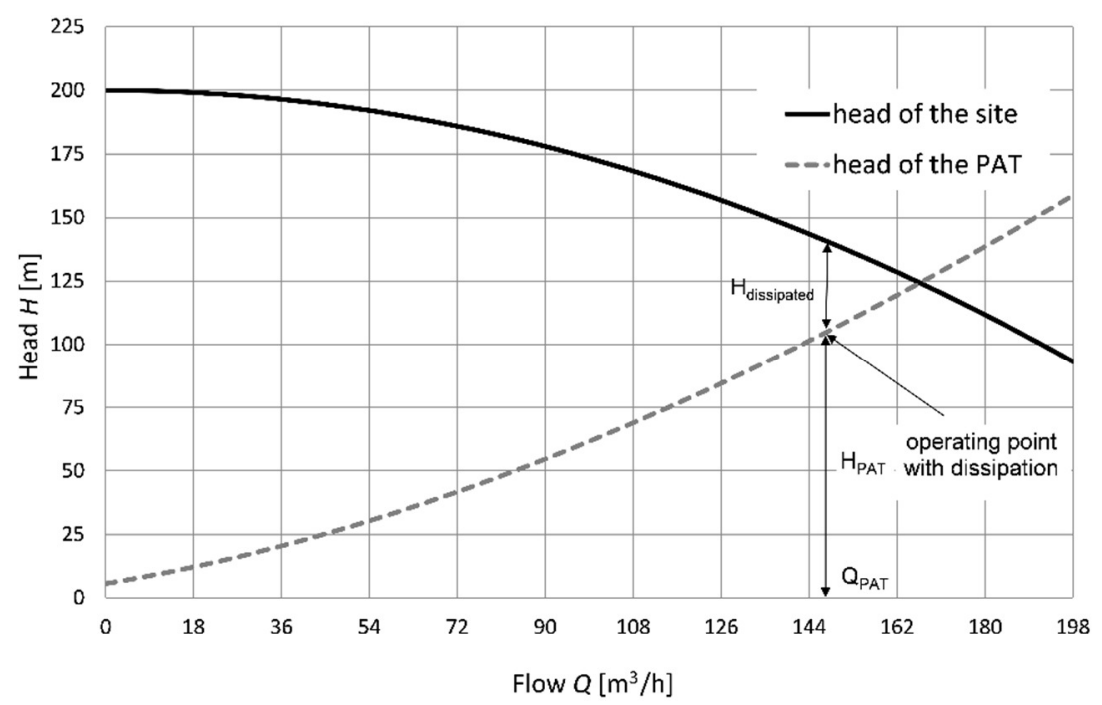

Figure 16. Operating point with flow rates lower than design value

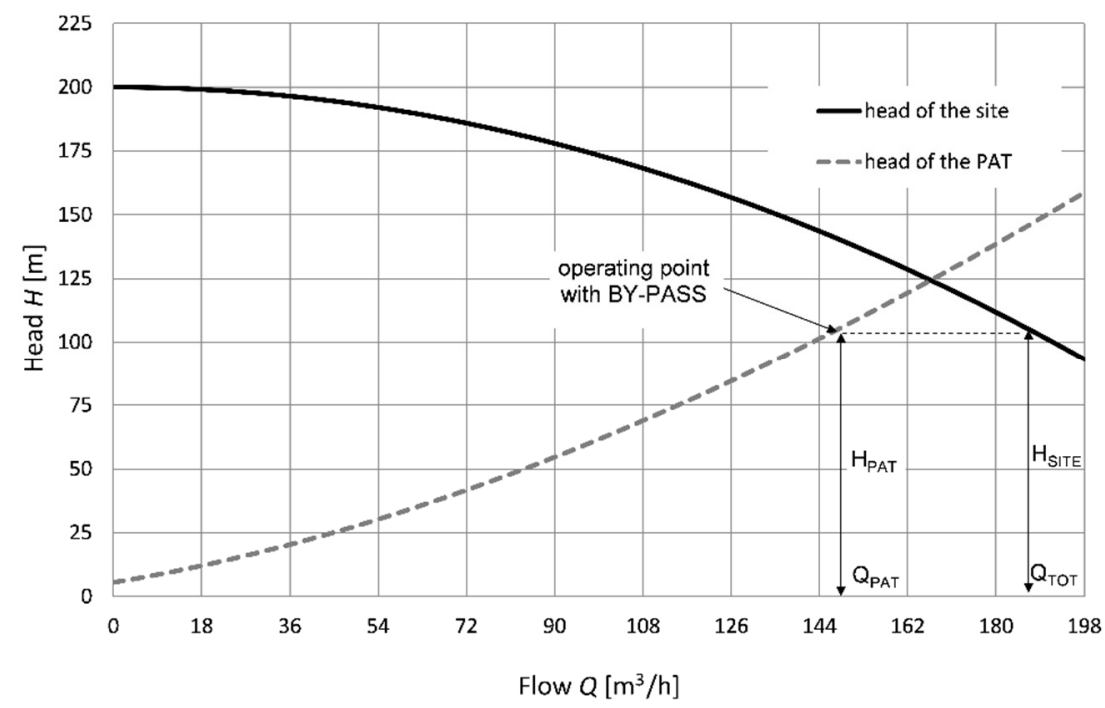

Figure 17. Operating point with flow rates higher than design value

Table 7. Yearly energy output of the PAT 65-250 for the Casali site

\begin{tabular}{cccccccc}
\hline$Q\left[\mathrm{~m}^{3} / \mathrm{h}\right]$ & $\begin{array}{c}\text { Frequencies } \\
\text { [hours/year] }\end{array}$ & $H_{\text {site }}[\mathrm{m}]$ & $Q_{\mathrm{T}}\left[\mathrm{m}^{3} / \mathrm{h}\right]$ & $H_{\mathrm{T}}[\mathrm{m}]$ & $\eta_{\mathrm{T}}$ & $\begin{array}{c}\text { Electrical power } \\
{[\mathrm{kW}]}\end{array}$ & $\begin{array}{c}\text { Energy output } \\
{[\mathrm{MWh} / \text { year }]}\end{array}$ \\
\hline 0.00 & 674 & 178.52 & 0.00 & 7.66 & 0.00 & 0.00 & 0.00 \\
142.20 & 533 & 139.39 & 89.31 & 113.90 & 0.630 & 16.75 & 8.94 \\
144.00 & 337 & 137.74 & 88.30 & 112.26 & 0.630 & 16.31 & 5.50 \\
145.80 & 84 & 136.07 & 87.28 & 110.59 & 0.629 & 15.87 & 1.34 \\
147.60 & 253 & 134.39 & 86.23 & 108.90 & 0.629 & 15.43 & 3.90 \\
149.40 & 225 & 132.68 & 85.16 & 107.19 & 0.628 & 14.99 & 3.37 \\
151.20 & 56 & 130.95 & 84.07 & 105.46 & 0.628 & 14.54 & 0.82 \\
153.00 & 28 & 129.20 & 82.96 & 103.71 & 0.627 & 14.10 & 0.40 \\
198.00 & 0 & 53.24 & 47.15 & 53.24 & 0.505 & 3.31 & 0.00 \\
199.80 & 0 & 50.95 & 45.35 & 50.95 & 0.483 & 2.92 & 0.00 \\
\hline Yearly hours & 8,760 & & & & Energy [MWh/year] & 123.06 \\
\hline
\end{tabular}


Table 8. Yearly energy output of the PAT 65-250 for the Sersale site

\begin{tabular}{cccccccc}
\hline$Q\left[\mathrm{~m}^{3} / \mathrm{h}\right]$ & $\begin{array}{c}\text { Frequencies } \\
\text { [hours/year] }\end{array}$ & $H_{\text {site }}[\mathrm{m}]$ & $Q_{\mathrm{T}}\left[\mathrm{m}^{3} / \mathrm{h}\right]$ & $H_{\mathrm{T}}[\mathrm{m}]$ & $\eta_{\mathrm{T}}$ & $\begin{array}{c}\text { Electrical power } \\
{[\mathrm{kW}]}\end{array}$ & $\begin{array}{c}\text { Energy output } \\
{[\mathrm{MWh} / \text { year }]}\end{array}$ \\
\hline 0.00 & 215 & 200.00 & 0.00 & 5.58 & 0.00 & 0.00 & 0.00 \\
162.00 & 762 & 128.55 & 162.00 & 119.47 & 0.703 & 35.59 & 27.11 \\
163.80 & 93 & 126.96 & 163.80 & 121.34 & 0.702 & 36.52 & 3.38 \\
165.60 & 469 & 125.34 & 165.60 & 123.21 & 0.702 & 37.46 & 17.57 \\
167.40 & 40 & 123.71 & 167.40 & 123.71 & 0.701 & 38.00 & 1.51 \\
169.20 & 203 & 122.06 & 164.28 & 122.06 & 0.700 & 36.72 & 7.45 \\
171.00 & 891 & 120.39 & 162.70 & 120.39 & 0.700 & 35.87 & 31.96 \\
172.80 & 1,044 & 118.71 & 161.09 & 118.71 & 0.695 & 34.77 & 36.30 \\
192.60 & 0 & 99.01 & 141.77 & 99.01 & 0.665 & 24.42 & 0.00 \\
194.40 & 0 & 97.11 & 139.84 & 97.11 & 0.665 & 23.63 & 0.00 \\
\hline Yearly hours & 8,760 & & & \multicolumn{3}{c}{ Energy [MWh/year] } & 283.25 \\
\hline
\end{tabular}

The following columns of the tables show the water purifier flow rates, the related frequencies, the heads available at the sites, the flow rates of the PAT, the heads of the PAT, the efficiencies of the PAT, the electrical powers and finally the energy outputs for each flow rate.

By summing all the energies related to the various frequencies the yearly energy of the PAT can be calculated, which in the present case is about $123 \mathrm{MWh}$ for the Casali site and $283 \mathrm{MWh}$ for the Sersale site by saving, respectively, 89 tonne of $\mathrm{CO}_{2} /$ year for Casali and 138 tonne of $\mathrm{CO}_{2}$ /year for Sersale.

By considering a state incentive for renewable energy of $0.2 \mathrm{EUR} / \mathrm{kWh}$ the intervention will yield about 25,000 EUR/year for Casali and 55,000 EUR/year for Sersale, thus providing payback times of less than a year since the estimated costs of installation (comprehensive of pump, inverter, manpower, valves, pipes and so on) are lower than the yearly revenues.

\section{CONCLUSIONS}

In the present work, the possibility of producing energy from small hydraulic sources has been analysed. A new methodology for selecting a PAT to be installed in a particular site is proposed. The method involves statistical models and a one-dimensional code: the statistical models allow calculating the conversion factors $C_{\mathrm{Q}}$ and $C_{\mathrm{H}}$, able to find capacity and flow rate of the suitable pump for the chosen site. By inputting these data on the composite performance chart of the manufacturer, the PAT can be selected. The UNICAL model developed by the authors will provide the performances curves head-capacity and efficiency-capacity.

The methodology has been applied to two study cases. Specifically, the technical-economic feasibility of the recovery interventions at the water purifier plants of Casali and Sersale has been considered. Here, the use of infrastructures already present, like penstock and intake, constitutes the principal advantage to do the installations.

The PAT selected for both the sites is 65-250 pump, able to provide about $123 \mathrm{MWh}$ for the Casali site and $283 \mathrm{MWh}$ for the Sersale site, by saving respectively 89 tonnes of $\mathrm{CO}_{2} /$ year and 138 tonnes of $\mathrm{CO}_{2} /$ year.

By considering a state incentive for renewable energy of $0.2 \mathrm{EUR} / \mathrm{kWh}$ the intervention will yield, about 25,000 EUR/year for Casali and 55,000 EUR/year for Sersale, providing payback times of less than a year.

The SoriCal company that manages the purifier plants, has expressed a strong interest to undertake the proposed interventions which will be achieved in the next few years.

\section{NOMENCLATURE}

$\begin{array}{llc}c & \text { velocity } & {[\mathrm{m} / \mathrm{s}]} \\ C_{\mathrm{H}} & \text { head conversion factor } & {[-]}\end{array}$




\begin{tabular}{llc}
\hline$C_{\mathrm{Q}}$ & flow rate conversion factor & {$[-]$} \\
$D_{2}$ & impeller diameter & {$[\mathrm{m}]$} \\
$D_{\mathrm{h}}$ & hydraulic diameter & {$[\mathrm{m}]$} \\
$g$ & gravity & {$\left[\mathrm{m} / \mathrm{sec}^{2}\right]$} \\
$h_{\mathrm{d}}$ & dynamic losses & {$[\mathrm{m}]$} \\
$h_{\mathrm{f}}$ & friction losses & {$[\mathrm{m}]$} \\
$H$ & head & {$[\mathrm{m}]$} \\
$H_{\mathrm{o}}$ & shut off head & {$[\mathrm{m}]$} \\
$H_{\mathrm{P}}$ & head of the PAT as pump & {$[\mathrm{m}]$} \\
$H_{\text {site }}$ & head of the site & {$[\mathrm{m}]$} \\
$H_{\mathrm{T}}$ & head of the PAT as turbine & {$[\mathrm{m}]$} \\
$l$ & duct length & {$[\mathrm{m}]$} \\
$n$ & rotational speed & {$[\mathrm{rpm}]$} \\
$n_{\mathrm{sp}}$ & specific speed of the PAT as pump & {$\left[\mathrm{rpm} \mathrm{m} \mathrm{m}^{3 / 4} \mathrm{~s}^{-1 / 2}\right]$} \\
$n_{\mathrm{st}}$ & specific speed of the PAT as turbine & {$\left[\mathrm{rpm} \mathrm{m} \mathrm{m}^{3 / 4} \mathrm{~s}^{-1 / 2}\right]$} \\
$N_{\text {site }}$ & specific speed of the site & {$\left[\mathrm{rpm} \mathrm{m} \mathrm{m}^{3 / 4} \mathrm{~s}^{-1 / 2}\right]$} \\
$P$ & power & {$[\mathrm{kW}]$} \\
$P_{\mathrm{el}}$ & electrical power & {$[\mathrm{kW}]$} \\
$P_{\mathrm{max}}$ & maximum power & {$[\mathrm{kW}]$} \\
$Q$ & flow rate & {$[1 / \mathrm{s}]$} \\
$Q_{\text {iteration }}$ & iterative value of flow rate & {$[1 / \mathrm{s}]$} \\
$Q_{\mathrm{P}}$ & flow rate of the PAT as pump & {$[1 / \mathrm{s}]$} \\
$Q_{\text {site }}$ & flow rate of the site & {$[1 / \mathrm{s}]$} \\
$Q_{\mathrm{T}}$ & flow rate of the PAT as turbine & {$[1 / \mathrm{s}]$} \\
$y_{\mathrm{p}}$ & pump height from axis to diffuser & {$[\mathrm{m}]$}
\end{tabular}

\section{Greek letters}

$\zeta$
$\eta_{\mathrm{D}}$
$\eta_{\mathrm{el}}$
$\eta_{\mathrm{H}}$
$\eta_{\mathrm{T}}$
$\eta_{\mathrm{v}}$
$\lambda$
$\rho$

dynamic loss coefficient

\section{REFERENCES}

1. Nastasi, B. and Lo Basso, G., Hydrogen to Link Heat and Electricity in the Transition towards Future Smart Energy Systems, Energy, Vol. 110, pp 5-22, 2016, https://doi.org/10.1016/j.energy.2016.03.097

2. Geller, M. T. B. and Meneses, A. A. d. M., Life Cycle assessment of a Small Hydropower Plant in the Brazilian Amazon, J. Sustain. Dev. Energy Water Environ. Syst., Vol. 4, No. 4, pp 379-391, 2016, https://doi.org/10.13044/j.sdewes.2016.04.0029

3. Williams, A. A., The Turbine Performance of Centrifugal Pumps: A comparison of prediction Methods, Proc. Instn. Mech. Engrs., Vol. 208, Pt. A, pp 59-66, 1994.

4. Motwani, K. H., Jain, S. V. and Patel, R. N., Cost analysis of Pump as Turbine for Pico Hydropower Plants - A Case Study, Procedia Engineering, Vol. 51, pp 721-726, 2013, https://doi.org/10.1016/j.proeng.2013.01.103

5. Hvala, N., Vrečko, D., Levstek, M. and Bordon, C., The use of Dynamic Mathematical Models for improving the Designs of upgraded Wastewater Treatment Plants, J. Sustain. Dev. Energy Water Environ. Syst., Vol. 5, No. 1, pp 15-31, 2017, https://doi.org/10.13044/j.sdewes.d5.0130 
6. Chapallaz, J. M., Eichenberger, P. and Fischer, G., Manual on Pumps used as Turbines, Informatica International, Incorporated, 1992.

7. Childs, S. M., Convert Pumps to Turbines and Recover HP, Hydrocarbon Processing an Petroleum Refiner, 1962.

8. Sharma, K. R., Small Hydroelectric Project-use of Centrifugal Pumps as Turbines, Kirloskar Electric Co., Bangalore, India, 1985.

9. Alatorre-Frenk, C. and Thomas, T. H., The Pumps as Turbines approach to Small Hydropower, Word Congress on Renewable energy, Reading, UK, 1990.

10. Stepanoff, A. J., Centrifugal and Axial Flow Pumps, John Wiley, New York, USA, pp 276, 1957.

11. Hancock, J. W., Centrifugal Pumps or Water Turbine, Pipe Line News, pp 25-27, 1963.

12. Schmield, E., Series of Centrifugal Pumps in Turbine Operation (in German), Pumpentagung Karlsruhe, Sec. A6, 1988.

13. Derakhshan, S. and Nourbakhsh, A., Experimental Study of Characteristic Curves of Centrifugal Pumps working as Turbines in different Specific Speeds, Experimental Thermal and Fluid Science, Vol. 32, No. 3, pp 800-807, 2008, https://doi.org/10.1016/j.expthermflusci.2007.10.004

14. Jain, S. V., Swarnkar, A., Motwani, K. H. and Patel, R. N., Effects of Impeller Diameter and Rotational Speed on Performance of Pump running in Turbine Mode, Energy Conversion and Management, Vol. 89, pp 808-824, 2015, https://doi.org/10.1016/j.enconman.2014.10.036

15. Amelio, M. and Barbarelli, S., A One-dimensional Numerical Model for calculating the efficiency of Pumps as Turbines for implementation in Micro-hydro Power Plants, Proceedings of " $7^{\text {th }}$ Biennial ASME Conference Engineering Systems Design and Analysis", Manchester, UK, July 19-22, 2004, https://doi.org/10.1115/ESDA2004-58080

16. Barbarelli, S., Amelio, M. and Florio, G., Predictive Model estimating the Performances of Centrifugal Pumps used as Turbines, Energy, Vol. 107, pp 103-121, 2016, https://doi.org/10.1016/j.energy.2016.03.122

17. Grover, K. M., Conversion of Pump to Turbines, GSA Inter Corp. Katonah, New York, USA, 1980.

18. Lewinsky-Kesslitz, H. P., Pumps as Turbines for Small Power Plants (in German), Wasserwirtschaft, Vol. 77, No. 10, pp 531-537, 1987.

19. Barbarelli, S., Amelio, M. and Florio, G., Experimental Activity at Test Rig validating Correlations to Select Pumps running as Turbines in Microhydro Plants, Energy Conversion and Management, Vol. 149, pp 781-797, 2017, https://doi.org/10.1016/j.enconman.2017.03.013

20. Williams, A. A., The Selection and Application of Centrifugal Pumps as Water Turbines, Proc. Mech. E, Vol. 208, Pt. A, pp 59-66, 1994.

21. Neumann, B., The interaction between Geometry and Performance of a Centrifugal Pump, Mechanical Engineering Publications Limited, London, UK, 1991.

22. Lobanoff, V. S. and Ross, R. R., Centrifugal Pumps \& design and Applications ( $2^{\text {nd }}$ ed.), Gulf Publishing Company, Houston, USA, 1992.

23. Idel'cick, E., Memento Load Loss, Eyrolles Edition, Paris, France, 1986. 